\title{
Traveling Waves for Delayed Cellular Neural Networks with Nonmonotonic Output Functions
}

\author{
Zhi-Xian Yu, ${ }^{1}$ Rong Yuan, ${ }^{2}$ Cheng-Hsiung Hsu, ${ }^{3}$ and Ming-Shu Peng ${ }^{4}$ \\ ${ }^{1}$ College of Science, University of Shanghai for Science and Technology, Shanghai 200093, China \\ ${ }^{2}$ School of Mathematical Sciences, Beijing Normal University, Beijing 100875, China \\ ${ }^{3}$ Department of Mathematics, National Central University, Jhongli 32001, Taiwan \\ ${ }^{4}$ Department of Mathematics, Beijing Jiaotong University, Beijing 100044, China
}

Correspondence should be addressed to Zhi-Xian Yu; zxyu0902@163.com

Received 13 April 2014; Accepted 25 June 2014; Published 22 July 2014

Academic Editor: Shengqiang Liu

Copyright (C) 2014 Zhi-Xian Yu et al. This is an open access article distributed under the Creative Commons Attribution License, which permits unrestricted use, distribution, and reproduction in any medium, provided the original work is properly cited.

\begin{abstract}
This work investigates traveling waves for a class of delayed cellular neural networks with nonmonotonic output functions on the one-dimensional integer lattice $\mathbb{Z}$. The dynamics of each given cell depends on itself and its nearest $m$ left or $l$ right neighborhood cells with distributed delay due to, for example, finite switching speed and finite velocity of signal transmission. Our technique is to construct two appropriate nondecreasing functions to squeeze the nonmonotonic output functions. Then we construct a suitable wave profiles set and derive the existence of traveling wave solutions by using Schauder's fixed point theorem.
\end{abstract}

\section{Introduction}

Recently, there have been an increasing activity and interest in the study of nervous systems, namely, in the study of equations modeling neural networks, which are applied to a broad scope of fields such as image and video signal processing, robotic and biological versions, and higher brain functions (see [1-6] for more details). The methodology of cellular neural networks (for short, CNN) was first proposed by Chua and Yang [7-9] as an achievable alternative to fully connected neural networks in electric circuit systems. The dynamic evolution of the network is governed by the assumed dynamics of the individual processing units and their reciprocal interactions. The infinite system of ordinary differential equations for $\mathrm{CNN}$ distributed in a one-dimensional integer lattice can be described by

$$
\begin{aligned}
\frac{d x_{n}(t)}{d t}= & -x_{n}(t)+z+\sum_{i=1}^{m} a_{i} f\left(x_{n-i}(t)\right)+\alpha f\left(x_{n}(t)\right) \\
& +\sum_{i=1}^{m} \beta_{i} f\left(x_{n+i}(t)\right),
\end{aligned}
$$

where $f$ is the piecewise-linear output function given by

$$
f(x)= \begin{cases}1, & \text { if } x \geq 1 \\ x, & \text { if }|x| \leq 1 \\ -1, & \text { if } x \leq-1\end{cases}
$$

The quantity $z$ is called a threshold or bias term and is related to independent voltage sources in electric circuits. The real constant coefficients $a_{i}$, $\alpha$, and $\beta_{i}$ of the output function $f$ constitute the so-called space-invariant template that measures the synaptic weights of self-feedback and neighborhood interaction. In recent years, when the output function $f$ is defined as (2), some incisive mathematical analyses have subsequently been done; see [1-3,7-9] and the references cited therein.

However, to be more realistic, the neural models should be incorporated into time delays, since the transmission of information from one neuron to another is not instantaneous. In models of electronic neural networks, the dynamics of each given cell depends on itself and its nearest left or right neighbors where delays exist in left or right neighborhood interactions due to, for example, finite switching speed and finite velocity of signal transmission (see [7]). Such 
assumptions can be also found in $[1,10-13]$. In the past decades, there are many mathematical results on the study of dynamics for neural models. Particularly, the investigation of traveling wave solutions has attracted much attention due to its significant nature in biology, chemistry, epidemiology, and physics, such as reaction-diffusion equations [14-23] and lattice equations $[2,8,24,25]$. For delayed CNN models (for short, DCNN) with the piecewise-linear output function (2), the existence of traveling waves has been investigated in $[1,11-$ 13]. If the output function is nonlinear, for example,

$$
f(x)= \begin{cases}1, & \text { if } x \geq 1 \\ \sin \frac{\pi}{2} x, & \text { if }|x| \leq 1 \\ -1, & \text { if } x \leq-1\end{cases}
$$

or

$$
f(x)= \begin{cases}1, & \text { if } x \geq 1 \\ 2 x-x^{2}, & \text { if } 0 \leq x \leq 1 \\ 2 x+x^{2}, & \text { if }-1 \leq x \leq 0 \\ -1, & \text { if } x \leq-1\end{cases}
$$

the authors of [10] also derived the existence of traveling wave solutions. Recently, when the propagation of signal is very slow, authors of [26] considered CNN models with infinite time delays. More precisely, Yu et al. [26] investigated the existence and nonexistence of monotonic traveling waves for the following more general $\mathrm{CNN}$ models with infinite distributed time delay terms:

$$
\begin{aligned}
x_{n}^{\prime}(t)= & -x_{n}(t)+\sum_{i=1}^{m} a_{i} \int_{0}^{\tau} J_{i}(y) f\left(x_{n-i}(t-y)\right) d y \\
& +\alpha \int_{0}^{\tau} J_{m+1}(y) f\left(x_{n}(t-y)\right) d y \\
& +\sum_{j=1}^{l} \beta_{j} \int_{0}^{\tau} J_{m+1+j}(y) f\left(x_{n+j}(t-y)\right) d y,
\end{aligned}
$$

for $n \in \mathbb{Z}, m, l \in \mathbb{N}$. Here $a_{i}(i=1, \ldots, m), \alpha$, and $\beta_{j}(j=1, \ldots, l)$ are nonnegative constants; $\tau$ may be a positive constant or infinity; and the output function $f(u)$ is nondecreasing. Here the dynamics of each given cell depends on itself and its nearest $m$ left or $l$ right neighborhood cells with distributed delay due to, for example, finite switching speed and finite velocity of signal transmission. Moreover, we point out that the CNN models with discrete time delays or no delays case can be included in this model by choosing suitable kernel functions (see [26]).

Note that some known results on the existence of traveling waves for (5) can be obtained only when the output functions are nondecreasing. But the output functions may not be nondecreasing. For example, let us consider the following two nonmonotonic output functions:

$$
\begin{gathered}
f(x)= \begin{cases}1, & \text { if } x \geq 1, \\
2 \sin \frac{5 \pi}{6} x, & \text { if }|x| \leq 1, \\
-1, & \text { if } x \leq-1,\end{cases} \\
f(x)= \begin{cases}1, & \text { if } x \geq 1, \\
3 x-2 x^{2}, & \text { if } 0 \leq x \leq 1, \\
3 x+2 x^{2}, & \text { if }-1 \leq x \leq 0, \\
-1, & \text { if } x \leq-1 .\end{cases}
\end{gathered}
$$

It is obvious that the previous results in $[2,3,10,12,27]$ cannot be applied for CNN or DCNN models with nonmonotonic output functions like (6) or (7). Therefore, it gives us the motivation to consider the existence of traveling wave solutions for DCNN model (5) with nonmonotonic output functions.

Throughout this paper, we assume that there exists a $b>0$ such that $f$ and $J$ satisfy the following assumptions.

(F1) $f \in C([0, b],[0, b /(a+\alpha+\beta)])$ is an odd function, $f(0)=0$, and there exist $K>0$ with $K \leq b$ and $L>0$ such that

$$
\begin{array}{r}
(a+\alpha+\beta) f(K)=K, \quad|f(u)-f(v)| \leq L|u-v|, \\
\text { for } u, v \in[0, b],
\end{array}
$$

where $a_{i} \geq 0, i=1, \ldots, m, \alpha \geq 0$, and $\beta_{j} \geq 0, j=1, \ldots, l$,

$$
a:=\sum_{i=1}^{m} a_{i}, \quad \beta:=\sum_{j=1}^{l} \beta_{j} .
$$

(F2) $f^{\prime}(0) u \geq f(u)>0,(a+\alpha+\beta) f(u)>u$ for $u \in(0, K)$, and $(a+\alpha+\beta) f(u)<u$ for $u \in(K, b]$.

(F3) There exists $\sigma \in(0,1]$ such that

$$
\limsup _{u \rightarrow 0^{+}}\left[f^{\prime}(0)-\frac{f(u)}{u}\right] u^{-\sigma}<+\infty .
$$

(F4) $(a+\alpha+\beta) f(u)<2 K-u$ for $u \in(0, K)$ and $(a+\alpha+$ $\beta) f(u)>2 K-u$ for $u \in(K, b]$.

$(J) J_{i}:[0, \tau] \rightarrow[0,+\infty)$ are piecewise continuous functions satisfying

$$
\int_{0}^{\tau} J_{i}(y) d y=1, \quad i=1, \ldots, m+l+1,
$$

where $0<\tau<\infty$. If $\tau=\infty$, we further assume that

$$
\begin{array}{r}
\int_{0}^{\infty} J_{i}(y) e^{\kappa y} d y<\infty \quad \text { for any } \kappa \in[0, \infty), \\
\\
i=1, \ldots, m+l+1 .
\end{array}
$$


Remark 1. If $f$ satisfies the following hypotheses,

(H1) $f$ is a continuous odd function, $f(0)=0, f(x)=1$ for $x \geq 1, \sum_{i=1}^{m} a_{i}+\sum_{j=1}^{l} \beta_{j}>0, \alpha f^{\prime}(0) \geq 1$, and there exists $L>0$ such that $|f(u)-f(v)| \leq L|u-v|$ for $u, v \in[0,1]$,

(H2) $f^{\prime}(0) u \geq f(u)>0$ for $u \in(0,1]$; in addition, $f^{\prime \prime}(0)$ exists and $(\alpha+a+\beta) f(u)>u$ for $u \in(0,1)$,

then it is obvious that (F1) $-(\mathrm{F} 3)$ hold and $0, K=\sum_{i=1}^{m} a_{i}+\alpha+$ $\sum_{j=1}^{l} \beta_{j}$, and $-K$ are three equilibria of (5).

Remark 2. If $a+\alpha+\beta>1$, then the nonmonotonic output functions (6) and (7) satisfy assumptions (F1)-(F4).

Our purpose is to investigate the existence of traveling waves for (5) with nonmonotonic output functions. A traveling wave solution of (5) is a special translation invariant solution of (12) with the form $x_{n}(t)=\phi(n-c t), i \in \mathbb{Z}$, and $t \in \mathbb{R}$ for a wave profile $\phi(\xi), \xi=n-c t \in \mathbb{R}$, with a given wave speed $c \in \mathbb{R}$. Letting $\xi=n-c t$, it follows that $\phi$ must be a solution of the following wave profile equation:

$$
\begin{aligned}
-c \phi^{\prime}(\xi)= & -\phi(\xi)+\sum_{i=1}^{m} a_{i} \int_{0}^{\tau} J_{i}(y) f(\phi(\xi-i+c y)) d y \\
& +\alpha \int_{0}^{\tau} J_{m+1}(y) f(\phi(\xi+c y)) d y \\
& +\sum_{j=1}^{l} \beta_{j} \int_{0}^{\tau} J_{m+1+j}(y) f(\phi(\xi+j+c y)) d y .
\end{aligned}
$$

Under assumptions (F1)-(F2), it is easily seen that 0 and $\pm K$ are three equilibria of (13). We are mainly interested in the existence of traveling waves satisfying one of the following asymptotic boundary conditions:

$$
\begin{array}{llc}
\text { (BC1) } & \lim _{\xi \rightarrow-\infty} \phi(\xi)=0, & \liminf _{\xi \rightarrow+\infty} \phi(\xi)>0 ; \\
\text { (BC2) } & \liminf _{\xi \rightarrow-\infty} \phi(\xi)>0, & \lim _{\xi \rightarrow+\infty} \phi(\xi)=0 ; \\
\text { (BC3) } & \lim _{\xi \rightarrow-\infty} \phi(\xi)=0, & \limsup _{\xi \rightarrow+\infty} \phi(\xi)<0 ; \\
\text { (BC4) } & \limsup _{\xi \rightarrow-\infty} \phi(\xi)<0, & \lim _{\xi \rightarrow+\infty} \phi(\xi)=0 .
\end{array}
$$

Since the output functions are nonmonotonic, our main idea is to construct two appropriate nondecreasing functions to squeeze the nonmonotonic output functions. Then we can apply the results in [26] and Schauder's fixed point theorem to derive the existence of traveling wave solutions. Now we state our main results.

Theorem 3. Assume (F1)-(F3) and (J) hold. If $\beta>0$ and $(\alpha+$ $\beta) f^{\prime}(0)>1$, then there exists $c_{1}^{*}<0$ such that the following statements hold.
(1) For each $c<c_{1}^{*}$, (5) has a traveling wave $\phi(n-c t)$ with speed $c$ such that $\phi \in C(\mathbb{R},[0, b]) /\{0, K\}$ and satisfies (BC1). Moreover,

$$
\begin{gathered}
\lim _{\xi \rightarrow+\infty} \phi(\xi)=K \\
\text { or } 0<\liminf _{\xi \rightarrow+\infty} \phi(\xi)<K<\limsup _{\xi \rightarrow+\infty} \phi(\xi) \leq b, \\
\lim _{\xi \rightarrow-\infty} \phi(\xi) e^{-\lambda_{1} \xi}=1, \quad \lim _{\xi \rightarrow-\infty} \phi^{\prime}(\xi) e^{-\lambda_{1} \xi}=\lambda_{1},
\end{gathered}
$$

where $\lambda_{1}$ is the smallest positive root of characteristic function of (13) at 0. If, in addition, (F4) holds, then $\lim _{\xi \rightarrow+\infty} \phi(\xi)=K$.

(2) For each $c<c_{1}^{*}$, (5) has a traveling wave $\psi(n-c t)$ with speed $c$ such that $\psi \in C(\mathbb{R},[-b, 0]) /\{0,-K\}$ and satisfies (BC3). Moreover,

$$
\begin{gathered}
\lim _{\xi \rightarrow+\infty} \psi(\xi)=-K \\
\text { or } \quad-b \leq \liminf _{\xi \rightarrow+\infty} \psi(\xi)<-K<\limsup _{\xi \rightarrow+\infty}(\xi)<0, \\
\lim _{\xi \rightarrow-\infty} \psi(\xi) e^{-\lambda_{1} \xi}=-1, \quad \lim _{\xi \rightarrow-\infty} \psi^{\prime}(\xi) e^{-\lambda_{1} \xi}=-\lambda_{1} .
\end{gathered}
$$

If, in addition, (F4) holds, then $\lim _{\xi \rightarrow+\infty} \psi(\xi)=-K$.

Theorem 4. Assume (F1)-(F3) and (J) hold. If $a>0$ and $(a+$ $\alpha) f^{\prime}(0)>1$, then there exists $c_{2}^{*}>0$ such that the following statements hold.

(1) For each $c>c_{2}^{*}$, (5) has a traveling wave $\phi(n-c t)$ with speed $c$ such that $\phi \in C(\mathbb{R},[0, b]) \backslash\{0, K\}$ and satisfies (BC2). Moreover,

$$
\begin{gathered}
\lim _{\xi \rightarrow-\infty} \phi(\xi)=K \\
\text { or } 0<\liminf _{\xi \rightarrow-\infty} \phi(\xi)<K<\limsup _{\xi \rightarrow-\infty}(\xi) \leq b, \\
\lim _{\xi \rightarrow+\infty} \phi(\xi) e^{-\lambda_{2} \xi}=1, \quad \lim _{\xi \rightarrow+\infty} \phi^{\prime}(\xi) e^{-\lambda_{2} \xi}=\lambda_{2},
\end{gathered}
$$

where $\lambda_{2}<0$ is the largest negative root of characteristic function of (13) at 0. If, in addition, (F4) holds, then $\lim _{\xi \rightarrow-\infty} \phi(\xi)=K$.

(2) For each $c>c_{2}^{*}$, (5) has a traveling wave $\psi(n-c t)$ with speed $c$ such that $\psi \in C(\mathbb{R},[-b, 0]) \backslash\{0,-K\}$ and satisfies (BC4). Moreover,

$$
\begin{gathered}
\lim _{\xi \rightarrow-\infty} \psi(\xi)=-K \\
\text { or } \quad-b \leq \liminf _{\xi \rightarrow-\infty} \psi(\xi)<-K<\limsup _{\xi \rightarrow-\infty}(\xi)<0, \\
\lim _{\xi \rightarrow+\infty} \psi(\xi) e^{-\lambda_{2} \xi}=-1, \quad \lim _{\xi \rightarrow+\infty} \psi^{\prime}(\xi) e^{-\lambda_{2} \xi}=-\lambda_{2} .
\end{gathered}
$$

If, in addition, (F4) holds, then $\lim _{\xi \rightarrow-\infty} \psi(\xi)=-K$. 
Theorem 5. Assume (F1)-(F3) and (J) hold. If $\beta=0$ and $(a+$ $\alpha) f^{\prime}(0)>1$, then the following statements hold.

(1) For any $c<0$, (5) has a traveling wave $\phi(n-c t)$ with speed $c$ such that $\phi \in C(\mathbb{R},[0, b]) \backslash\{0, K\}$ and satisfies (BC3). Moreover,

$$
\begin{gathered}
\lim _{\xi \rightarrow+\infty} \phi(\xi)=K \\
\text { or } \quad 0<\liminf _{\xi \rightarrow+\infty} \phi(\xi)<K<\limsup _{\xi \rightarrow+\infty} \phi(\xi) \leq b, \\
\lim _{\xi \rightarrow-\infty} \phi(\xi) e^{-\lambda_{3} \xi}=1, \quad \lim _{\xi \rightarrow-\infty} \phi^{\prime}(\xi) e^{-\lambda_{3} \xi}=\lambda_{3},
\end{gathered}
$$

where $\lambda_{3}>0$ is a unique real root of characteristic function of (13) at 0. If, in addition, (F4) holds, then $\lim _{\xi \rightarrow+\infty} \phi(\xi)=K$.

(2) For any $c<0$, (5) has a traveling wave $\psi(n-c t)$ with speed $c$ such that $\psi \in C(\mathbb{R},[-b, 0]) \backslash\{0,-K\}$ and satisfies (BC3). Moreover,

$$
\begin{gathered}
\lim _{\xi \rightarrow+\infty} \psi(\xi)=-K \\
\text { or } \quad-b \leq \liminf _{\xi \rightarrow+\infty} \psi(\xi)<-K<\limsup _{\xi \rightarrow+\infty}(\xi)<0, \\
\lim _{\xi \rightarrow-\infty} \psi(\xi) e^{-\lambda_{3} \xi}=-1, \quad \lim _{\xi \rightarrow-\infty} \psi^{\prime}(\xi) e^{-\lambda_{3} \xi}=-\lambda_{3} .
\end{gathered}
$$

If, in addition, (F4) holds, then $\lim _{\xi \rightarrow+\infty} \psi(\xi)=-K$.

Theorem 6. Assume (F1)-(F3) and (J) hold. If $a=0$ and $(\alpha+$ $\beta) f^{\prime}(0)>1$, then the following statements hold.

(1) For any $c>0$, (5) has a traveling wave $\phi(n-c t)$ with speed $c$ such that $\phi \in C(\mathbb{R},[0, b]) \backslash\{0, K\}$ and satisfies (BC2). Moreover,

$$
\begin{gathered}
\lim _{\xi \rightarrow-\infty} \phi(\xi)=K \\
\text { or } \quad 0<\liminf _{\xi \rightarrow-\infty} \phi(\xi)<K<\limsup _{\xi \rightarrow-\infty} \phi(\xi) \leq b, \\
\lim _{\xi \rightarrow+\infty} \phi(\xi) e^{-\lambda_{4} \xi}=1, \quad \lim _{\xi \rightarrow+\infty} \phi^{\prime}(\xi) e^{-\lambda_{4} \xi}=\lambda_{4},
\end{gathered}
$$

where $\lambda_{4}<0$ is a unique real root of characteristic function of (13) at 0. If, in addition, (F4) holds, then $\lim _{\xi \rightarrow-\infty} \phi(\xi)=K$.

(2) For any $c>0,(5)$ has a traveling wave $\psi(n-c t)$ with speed $c$ such that $\psi \in C(\mathbb{R},[-b, 0]) \backslash\{0,-K\}$ satisfies (BC4). Moreover,

$$
\begin{gathered}
\lim _{\xi \rightarrow-\infty} \psi(\xi)=-K \\
\text { or } \quad-b \leq \liminf _{\xi \rightarrow-\infty} \psi(\xi)<-K<\limsup _{\xi \rightarrow-\infty}(\xi)<0, \\
\lim _{\xi \rightarrow+\infty} \psi(\xi) e^{-\lambda_{4} \xi}=-1, \quad \lim _{\xi \rightarrow+\infty} \psi^{\prime}(\xi) e^{-\lambda_{4} \xi}=-\lambda_{4} .
\end{gathered}
$$

If, in addition, (F4) holds, then $\lim _{\xi \rightarrow-\infty} \psi(\xi)=-K$.
Remark 7. In the above theorems, we obtain that the traveling waves either converge to the nontrivial equilibrium $(K$ or $-K)$ or oscillate on the nontrivial equilibrium at infinity. Furthermore, we give a sufficient condition for the convergence of traveling waves to the nontrivial equilibrium. But under what conditions the wave will oscillate on the nontrivial equilibrium at infinity is an interesting problem. On the other hand, similarly to the proofs of nonexistence of traveling waves in [26], we can also obtain the same conclusions for nonmonotonic DCNN models.

Remark 8. The different results can be obtained through the choice of the signs for $a$ and $\beta$ and (5) reduces to kinds of CNN model with mixed delays by choosing suitable kernel functions. Please refer to [26].

The remainder of this paper is organized as follows. In Section 2, we recall some properties of the characteristic function of (13). Then, in Section 3, we construct two appropriate nondecreasing functions to squeeze the nonmonotonic output functions. Based on the construction of two nondecreasing functions, we devote Section 4 to proofs of the existence of traveling waves for (5). Our approach is to squeeze the nonmonotonic output functions and Schauder's fixed point theorem in a suitable Banach space. Furthermore, according to the construction of a wave profiles set, we can obtain the exponential asymptotic behavior of traveling waves in the infinity.

\section{Properties of the Characteristic Function}

In this section, we recall some properties of the characteristic function for (13). The characteristic function arises from the linearized equation of (13) at the equilibrium solution 0 and is given by

$$
\begin{aligned}
\Delta(\lambda, c):= & -c \lambda+1-f^{\prime}(0) \sum_{i=1}^{m} a_{i} \int_{0}^{\tau} J_{i}(y) e^{\lambda(-i+c y)} d y \\
& -f^{\prime}(0) \alpha \int_{0}^{\tau} J_{m+1}(y) e^{\lambda c y} d y \\
& -f^{\prime}(0) \sum_{j=1}^{l} \beta_{j} \int_{0}^{\tau} J_{m+1+j}(y) e^{\lambda(j+c y)} d y .
\end{aligned}
$$

The main properties of the characteristic function are stated in the following lemmas, and all the proofs can be found in our recent work [26].

Lemma 9. Assume that $\beta>0$ and $(\alpha+\beta) f^{\prime}(0)>1$. Then there exists a unique $c_{1}^{*}<0$ such that

(1) if $c \leq c_{1}^{*}$, then there exist two positive numbers $\lambda_{1}(c)$ and $\hat{\lambda}_{1}(c)$ with $\lambda_{1}:=\lambda_{1}(c) \leq \hat{\lambda}_{1}:=\hat{\lambda}_{1}(c)$ such that $\Delta\left(\lambda_{1}, c\right)=\Delta\left(\widehat{\lambda}_{1}, c\right)=0$; 
(2) if $c=c_{1}^{*}$, then $\lambda_{1}=\hat{\lambda}_{1}=\lambda_{1}^{*}$, and if $c<c_{1}^{*}$, then $\lambda_{1}<\lambda_{1}^{*}<\widehat{\lambda}_{1}$

$$
\begin{gathered}
\Delta(\cdot, c)>\quad \text { in }\left(\lambda_{1}, \hat{\lambda}_{1}\right), \\
\Delta(\cdot, c)<\quad \text { in } \mathbb{R} \backslash\left[\lambda_{1}, \hat{\lambda}_{1}\right] ;
\end{gathered}
$$

(3) if $c>c_{1}^{*}$, then $\Delta(\lambda, c)<0$ for all $\lambda \geq 0$.

Lemma 10. Assume that $a>0$ and $(a+\alpha) f^{\prime}(0)>1$. Then there exists a unique $c_{2}^{*}>0$ such that

(1) if $c \geq c_{2}^{*}$, then there exist $\lambda_{2}<0$ and $\hat{\lambda}_{2}<0$ with $\Delta\left(\lambda_{2}, c\right)=\Delta\left(\widehat{\lambda}_{2}, c\right)=0$

(2) if $c=c_{2}^{*}$, then $\lambda_{2}=\hat{\lambda}_{2}=\lambda_{2}^{*}$, and if $c>c_{2}^{*}$, then $\widehat{\lambda}_{2}<\lambda_{2}^{*}<\lambda_{2}$,

$$
\begin{gathered}
\Delta(\cdot, c)>\quad \text { in }\left(\hat{\lambda}_{2}, \lambda_{2}\right), \\
\Delta(\cdot, c)<\quad \text { in } \mathbb{R} \backslash\left[\hat{\lambda}_{2}, \lambda_{2}\right] ;
\end{gathered}
$$

(3) if $c<c_{2}^{*}$, then $\Delta(\lambda, c)<0$ for all $\lambda \leq 0$.

Lemma 11. Assume that $\beta=0$ and $(a+\alpha) f^{\prime}(0)>1$. Then we have the following:

(1) for any $c<0$, there exists a unique real root $\lambda_{3}:=$ $\lambda_{3}(c)>0$ such that $\Delta\left(\lambda_{3}, c\right)=0$. Moreover, for $c<0$, there exists $\bar{\epsilon}>0$ such that $\Delta\left(\lambda_{3}+\epsilon, c\right)>0$ for $\epsilon \in(0, \bar{\epsilon})$ and $\Delta(\lambda, c)>0$ for $\lambda \in\left(\lambda_{3}, \lambda_{3}+\epsilon\right)$;

(2) for $c \geq 0, \Delta(\lambda, c)<0$ for all $\lambda>0$.

Lemma 12. Assume that $a=0$ and $(\alpha+\beta) f^{\prime}(0)>1$. Then we have the following:

(1) for any $c>0$, there exists a unique real root $\lambda_{4}:=$ $\lambda_{4}(c)<0$ such that $\Delta\left(\lambda_{4}, c\right)=0$. Moreover, for any $c>0$, there exists $\widehat{\epsilon}>0$ such that $\Delta\left(\lambda_{4}-\epsilon, c\right)>0$ for $\epsilon \in(0, \widehat{\epsilon})$ and $\Delta(\lambda, c)>0$ for $\lambda \in\left(\lambda_{4}-\epsilon, \lambda_{4}\right)$;

(2) for $c \leq 0, \Delta(\lambda, c)<0$ for all $\lambda<0$.

According to the results of the above lemmas, in Section 4, we can see that the roots of characteristic equation play crucial roles in studying the behavior solutions of (13) near the equilibrium 0 .

\section{Construction of Nondecreasing Functions}

In this section, we will construct two nondecreasing functions such that $f$ lies between the two functions. Based on the construction of the nondecreasing functions, we can apply the results in [26] to derive the existence results.

Let us define functions $f^{-}(u)$ and $f^{+}(u)$ by

$$
\begin{aligned}
& f^{-}(u)=\inf _{u \leq v \leq b}\{f(v)\} \\
& f^{+}(u)=\min \left\{f^{\prime}(0) u, \frac{b}{a+\alpha+\beta}\right\} \\
& \text { for } u \in[0, b] .
\end{aligned}
$$

Then $f^{-}(u)$ and $f^{+}(u)$ satisfy the following properties.
Lemma 13. Assume that (F1)-(F3) hold.

(1) $f^{-}(u)$ and $f^{+}(u)$ are nondecreasing and Lipschitz continuous on $[0, b]$.

(2) $f^{-}(u) \leq f(u) \leq f^{+}(u)$ for all $u \in[0, b]$.

(3) $f^{\prime}(0) u \geq f^{-}(u)>0$ and $f^{\prime}(0) u \geq f^{+}(u)>0$ for all $u \in(0, b]$.

(4) $f^{+}(0)=(a+\alpha+\beta) f^{+}(b)-b=0$ and there exists an $u_{-}^{*}$ with $0<u_{-}^{*} \leq K$ such that $f^{-}(0)=(a+\alpha+\beta) f^{-}\left(u_{-}^{*}\right)-$ $u_{-}^{*}=0$. Moreover, we have $(a+\alpha+\beta) f^{-}(u)>u$ for any $u \in\left(0, u_{-}^{*}\right)$ and $(a+\alpha+\beta) f^{+}(u)>u$ for any $u \in(0, b)$.

(5) There exists $\sigma \in(0,1]$ such that

$$
\limsup _{u \rightarrow 0^{+}}\left[f^{\prime}(0)-\frac{f^{-}(u)}{u}\right] u^{-\sigma}<\infty \text {. }
$$

Proof. It is obvious that the assertions of (1), (3), and (5) hold. Therefore, we only prove the assertions of parts (2) and (4).

(2) By the definition of $f^{-}(u)$ and (F2), we have $f^{-}(u) \leq$ $f(u)$ for $u \in[0, b]$. According to (F1)-(F2), it is obvious that $f(u) \leq f^{\prime}(0) u$ and $f(u) \leq b /(a+\alpha+\beta)$ for $u \in[0, b]$. Thus, $f(u) \leq f^{+}(u)$ for $u \in[0, b]$.

(4) Obviously, $f^{-}(0)=f^{+}(0)=0$. Since $(a+\alpha+\beta) f^{\prime}(0)>$ 1 , it follows that $(a+\alpha+\beta) f^{+}(b)=b$ and there exists $\kappa>0$ such that $(a+\alpha+\beta) f^{-}(\kappa)>\kappa$. By the assertion of part (2), we have

$$
(a+\alpha+\beta) f^{-}(K) \leq(a+\alpha+\beta) f(K)=K .
$$

Thus, there exists an $u_{-}^{*} \in(\kappa, K]$ such that

$$
\begin{array}{r}
(a+\alpha+\beta) f^{-}\left(u_{-}^{*}\right)=u_{-}^{*}, \quad(a+\alpha+\beta) f^{-}(u)>u \\
\text { for any } u \in\left(0, u_{-}^{*}\right) .
\end{array}
$$

On the other hand, since $(a+\alpha+\beta) f^{\prime}(0) u>u$ and $b>u$ for any $u \in(0, b)$, by the definition of $f^{+}(u)$, we have $(a+\alpha+$ $\beta) f^{+}(u)>u$ for any $u \in(0, b)$. This completes the proof.

\section{Existence of Traveling Wave Solutions}

Now, applying the techniques developed in [26] to the functions $f^{-}(u)$ and $f^{+}(u)$, we prove the main theorems in the sequel.

4.1. Traveling Waves with (BC1) and (BC3). First, we consider the existence of traveling wave solutions satisfying condition (BC1). Once there exist traveling waves with (BC1), then we can easily obtain that (5) admits traveling wave solutions satisfying condition (BC3). by

Let us define the operator $T: C(\mathbb{R},[0, b]) \rightarrow C(\mathbb{R},[0, b])$

$$
T(\phi)(\xi)=e^{-\gamma \xi} \int_{-\infty}^{\xi} e^{\gamma y} F(\phi)(y) d y,
$$


where $\gamma>-1 / c>0$ is a constant and

$$
\begin{aligned}
F(\phi)(\xi)= & \left(\gamma+\frac{1}{c}\right) \phi(\xi) \\
& -\frac{1}{c} \sum_{i=1}^{m} a_{i} \int_{0}^{\tau} J_{i}(y) f(\phi(\xi-i+c y)) d y \\
& -\frac{\alpha}{c} \int_{0}^{\tau} J_{m+1}(y) f(\phi(\xi+c y)) d y \\
& -\frac{1}{c} \sum_{j=1}^{l} \beta_{j} \int_{0}^{\tau} J_{m+1+j}(y) f(\phi(\xi+j+c y)) d y .
\end{aligned}
$$

According to (F1) and $c<0, T$ is well defined. It is easy to see that a fixed point $\phi$ of $T$ or a solution of the equation

$$
\phi(\xi)=T(\phi)(\xi), \quad \xi \in \mathbb{R},
$$

is a traveling wave solution of (5).

Let $T^{ \pm}$be defined as in (30) with $f$ replaced by $f^{ \pm}$; that is,

$$
T^{ \pm}(\phi)(\xi)=e^{-\gamma \xi} \int_{-\infty}^{\xi} e^{\gamma y} F^{ \pm}(\phi)(y) d y,
$$

where

$$
\begin{aligned}
F^{ \pm}(\phi)(\xi)= & \left(\gamma+\frac{1}{c}\right) \phi(\xi) \\
& -\frac{1}{c} \sum_{i=1}^{m} a_{i} \int_{0}^{\tau} J_{i}(y) f^{ \pm}(\phi(\xi-i+c y)) d y \\
& -\frac{\alpha}{c} \int_{0}^{\tau} J_{m+1}(y) f^{ \pm}(\phi(\xi+c y)) d y \\
& -\frac{1}{c} \sum_{j=1}^{l} \beta_{j} \int_{0}^{\tau} J_{m+1+j}(y) f^{ \pm}(\phi(\xi+j+c y)) d y .
\end{aligned}
$$

According to Lemma 13, it is easily seen that $F^{ \pm}$and $T^{ \pm}$are nondecreasing on $C(\mathbb{R},[0, b])$ and

$$
T^{-}(\phi) \leq T(\phi) \leq T^{+}(\phi), \quad \text { for } \phi \in C(\mathbb{R},[0, b]) .
$$

Now we define the functions $\bar{\phi}^{+}(\xi)$ and $\bar{\phi}^{-}(\xi)$ by

$$
\begin{array}{r}
\bar{\phi}^{+}(\xi)=: \min \left\{b, e^{\lambda_{1} \xi}\right\}, \quad \bar{\phi}^{-}(\xi)=: \min \left\{u_{-}^{*}, e^{\lambda_{1} \xi}\right\} \\
\text { for } \xi \in \mathbb{R} .
\end{array}
$$

Since $u_{-}^{*} \leq b$, it is obvious that $\bar{\phi}^{-}(\xi) \leq \bar{\phi}^{+}(\xi)$. Similar to the proof of Lemma 3.1 in [26], it is easily seen that the following lemma holds.

Lemma 14. Assume that (F1)-(F3) hold. Then, for any $c<c_{1}^{*}$,

$$
T^{+}\left(\bar{\phi}^{+}\right)(\xi) \leq \bar{\phi}^{+}(\xi), \quad T^{-}\left(\bar{\phi}^{-}\right)(\xi) \leq \bar{\phi}^{-}(\xi), \quad \forall \xi \in \mathbb{R} .
$$

Since $f^{-}$is nondecreasing and satisfies the assumptions illustrated in [26], then we obtain the following result.

Lemma 15. Assume that (F1)-(F3) hold. Then, for any $c<c_{1}^{*}$, there exists a fixed point $\phi^{-}$of $T^{-}$such that $T^{-}\left(\phi^{-}\right)(\xi)=\phi^{-}(\xi)$ and $\phi^{-}(\xi) \leq \bar{\phi}^{-}(\xi)$ for all $\xi \in \mathbb{R}$. Moreover,

$$
\lim _{\xi \rightarrow-\infty} \phi^{-}(\xi) e^{-\lambda_{1} \xi}=1, \quad \lim _{\xi \rightarrow+\infty} \phi^{-}(\xi)=u_{-}^{*}
$$

Proof. The proof is the same as that of Theorem 1.1 of [26], and it is omitted.

By Lemmas 13-15 and (33), we easily verify that

$$
\begin{aligned}
\phi^{-}(\xi) & =T^{-}\left(\phi^{-}\right)(\xi) \leq T^{-}\left(\bar{\phi}^{-}\right)(\xi) \leq T^{+}\left(\bar{\phi}^{-}\right)(\xi) \\
& \leq T^{+}\left(\bar{\phi}^{+}\right)(\xi) \leq \bar{\phi}^{+}(\xi) .
\end{aligned}
$$

For a given number $\lambda>0$, let

$$
\begin{gathered}
X_{\lambda}:=\left\{\phi \in C(\mathbb{R}, \mathbb{R})\left|\sup _{\xi \in \mathbb{R}}\right| \phi(\xi) \mid e^{-\lambda \xi}<\infty\right\}, \\
\|\phi\|_{X_{\lambda}}=\sup _{\xi \in \mathbb{R}}|\phi(\xi)| e^{-\lambda \xi} .
\end{gathered}
$$

It is easy to see that $\left(X_{\lambda},\|\cdot\|_{X_{\lambda}}\right)$ is a Banach space. Then we define the following set:

$$
\Gamma:=\left\{\phi \in X_{\lambda} \mid \phi^{-}(\xi) \leq \phi(\xi) \leq \bar{\phi}^{+}(\xi), \text { for } \xi \in \mathbb{R}\right\}
$$

where $\lambda \in\left(0, \lambda_{1}\right)$. By (39) and the definitions of $\phi^{-}, \bar{\phi}^{+}$, we easily check that $\Gamma$ is a nonempty closed convex subset of $X_{\lambda}$. Thus, we have the following assertion.

Lemma 16. Assume that (F1)-(F2) hold. Then we have

(1) $T(\Gamma) \subset \Gamma$;

(2) $T: \Gamma \rightarrow \Gamma$ is completely continuous with respect to the norm $\|\cdot\|_{X_{\lambda}}$ in $X_{\lambda}$.

Proof. (1) For $\phi \in \Gamma$, by (39) and Lemmas 14-15, it follows that

$$
\begin{aligned}
\phi^{-}(\xi) & =T^{-}\left(\phi^{-}\right)(\xi) \leq T^{-}(\phi)(\xi) \leq T(\phi)(\xi) \\
& \leq T^{+}(\phi)(\xi) \leq T^{+}\left(\bar{\phi}^{+}\right)(\xi) \leq \bar{\phi}^{+}(\xi),
\end{aligned}
$$

which implies that $T(\Gamma) \subset \Gamma$.

(2) For any $\phi, \psi \in \Gamma$, it follows from (F1) that

$|F(\phi)(\xi)-F(\psi)(\xi)|$

$$
\leq\left(\gamma+\frac{1}{c}\right)|\phi(\xi)-\psi(\xi)|
$$




$$
\begin{aligned}
& -\frac{\alpha}{c} \int_{0}^{\tau} J_{m+1}(y)|f(\phi(\xi+c y))-f(\psi(\xi+c y))| d y \\
& -\frac{1}{c} \sum_{i=1}^{m} a_{i} \int_{0}^{\tau} J_{i}(y) \\
& \quad \times|f(\phi(\xi-i+c y))-f(\psi(\xi-i+c y))| d y \\
& -\frac{1}{c} \sum_{j=1}^{l} \beta_{j} \int_{0}^{\tau} J_{m+1+j}(y) \\
& \leq\left(\gamma+\frac{1}{c}\right)|\phi(\xi)-\psi(\xi)| \\
& -\frac{L \alpha}{c} \int_{0}^{\tau} J_{m+1}(y)|\phi(\xi+c y)-\psi(\xi+c y)| d y \\
& -\frac{L}{c} \sum_{i=1}^{m} a_{i} \int_{0}^{\tau} J_{i}(y)|\phi(\xi-i+c y)-\psi(\xi-i+c y)| d y \\
& -\frac{L}{c} \sum_{j=1}^{l} \beta_{j} \int_{0}^{\tau} J_{m+1+j}(y) \\
& \times(\xi+j+c y))-f(\psi(\xi+j+c y)) \mid d y \\
& \times(\xi+j+c y)-\psi(\xi+j+c y) \mid d y .
\end{aligned}
$$

Thus, we have

$$
\begin{gathered}
\|T(\phi)-T(\psi)\|_{X_{\lambda}} \\
=\sup _{\xi \in \mathbb{R}}|T(\phi)(\xi)-T(\psi)(\xi)| e^{-\lambda \xi} \\
\leq \sup _{\xi \in \mathbb{R}} e^{-(\gamma+\lambda) \xi} \int_{-\infty}^{\xi}|F(\phi)(s)-F(\psi)(s)| e^{\gamma s} d s \\
\leq \frac{1}{\lambda+\gamma}\left[\gamma+\frac{1}{c}-\frac{L}{c} \sum_{i=1}^{m} a_{i} \int_{0}^{\tau} J_{i}(y) e^{\lambda(c y-i)} d y\right. \\
\quad-\frac{L \alpha}{c} \int_{0}^{\tau} J_{m+1}(y) e^{c \lambda y} d y \\
\left.\quad-\frac{L}{c} \sum_{j=1}^{l} \beta_{j} \int_{0}^{\tau} J_{m+1+j}(y) e^{\lambda(c y+j)} d y\right] \\
\times\|\phi-\psi\|_{X_{\lambda}}{ }^{\tau}
\end{gathered}
$$

which implies that $T: \Gamma \rightarrow \Gamma$ is continuous. On the other hand, for any $\phi \in \Gamma, \xi \in \mathbb{R}$, we have $F(\phi)(\xi) \leq \gamma b$, and

$$
\begin{aligned}
& \left|T(\phi)\left(\xi_{1}\right)-T(\phi)\left(\xi_{2}\right)\right| \\
& \quad \leq \gamma b\left[e^{-\gamma \xi_{1}} \int_{-\infty}^{\xi_{1}} e^{\gamma s} d s-e^{-\gamma \xi_{2}} \int_{-\infty}^{\xi_{2}} e^{\gamma s} d s\right]
\end{aligned}
$$

$$
\begin{aligned}
& \leq \gamma b\left[e^{-\gamma \xi_{1}} \int_{\xi_{2}}^{\xi_{1}} e^{\gamma s} d s+\left|e^{-\gamma \xi_{2}}-e^{-\gamma \xi_{1}}\right| \int_{-\infty}^{\xi_{2}} e^{\gamma s} d s\right] \\
& =2 b\left[1-e^{-\gamma\left(\xi_{1}-\xi_{2}\right)}\right]
\end{aligned}
$$

for $\xi_{1} \geq \xi_{2}, \xi_{1}, \xi_{2} \in \mathbb{R}$. Since $\lim _{\xi \rightarrow 0}\left(1-e^{-\gamma \xi}\right)=0$, it follows that the family of functions $\{T(\phi)(\xi): \phi \in \Gamma\}$ is uniformly bounded and equicontinuous in $\xi \in \mathbb{R}$. Thus, by Arzela-Ascoli theorem, for any given sequence $\left\{\psi_{n}\right\}_{n \in \mathbb{N}^{+}}$ in $T(\Gamma)$, there exist $n_{k} \rightarrow \infty$ and $\psi \in C(\mathbb{R}, \mathbb{R})$ such that $\lim _{k \rightarrow \infty} \psi_{n_{k}}(\xi)=\psi(\xi)$ uniformly for $\xi$ in any compact subset of $\mathbb{R}$. Since $\phi^{-}(\xi) \leq \psi_{n_{k}}(\xi) \leq \bar{\phi}^{+}(\xi)$ for any $\xi \in \mathbb{R}$, we have $\phi^{-}(\xi) \leq \psi(\xi) \leq \bar{\phi}^{+}(\xi)$ for any $\xi \in \mathbb{R}$, and therefore $\psi(\xi) \in \Gamma$.

Next, we claim that

$$
\lim _{\xi \rightarrow \pm \infty}\left(\bar{\phi}^{+}(\xi)-\phi^{-}(\xi)\right) e^{-\lambda \xi}=0 .
$$

In fact, since $\lim _{\xi \rightarrow+\infty} \bar{\phi}^{+}(\xi)=b$ and $\lim _{\xi \rightarrow+\infty} \phi^{-}(\xi)=u_{*}^{-}$, we have

$$
\begin{aligned}
& \lim _{\xi \rightarrow+\infty}\left(\bar{\phi}^{+}(\xi)-\phi^{-}(\xi)\right) e^{-\lambda \xi} \\
& \quad=\lim _{\xi \rightarrow+\infty} \bar{\phi}^{+}(\xi) e^{-\lambda \xi}-\lim _{\xi \rightarrow+\infty} \phi^{-}(\xi) e^{-\lambda \xi}=0 .
\end{aligned}
$$

Since $\lim _{\xi \rightarrow-\infty} \phi^{-}(\xi) e^{-\lambda_{1} \xi}=1$ and $0<\lambda<\lambda_{1}$, it follows that

$$
\begin{aligned}
& \lim _{\xi \rightarrow-\infty}\left(\bar{\phi}^{+}(\xi)-\phi^{-}(\xi)\right) e^{-\lambda \xi} \\
& \quad=\lim _{\xi \rightarrow-\infty} e^{\left(\lambda_{1}-\lambda\right) \xi}-\lim _{\xi \rightarrow-\infty} \phi^{-}(\xi) e^{-\lambda_{1} \xi} e^{\left(\lambda_{1}-\lambda\right) \xi}=0 .
\end{aligned}
$$

Thus, it is easy to see that $T: \Gamma \rightarrow \Gamma$ is compact with respect to the norm $\|\cdot\|_{X_{\lambda}}$ in $X_{\lambda}$. The proof is complete.

Now we start the proofs of Theorems 3 and 5.

Proof of Theorem 3. (1) By Lemma 3.4, Schauder's fixed point theorem implies that there exists $\phi \in \Gamma$ such that $\phi=T(\phi)$. Since

$$
0 \leq \phi^{-}(\xi) \leq \phi(\xi) \leq \bar{\phi}^{+}(\xi) \leq b \quad \text { for } \xi \in \mathbb{R},
$$

we can easily obtain that, for $c<c_{1}^{*}$,

$$
\begin{gathered}
\lim _{\xi \rightarrow-\infty} \phi(\xi)=0, \\
\lim _{\xi \rightarrow-\infty} \phi(\xi) e^{-\lambda_{1} \xi}=1, \\
\lim _{\xi \rightarrow-\infty} \phi^{\prime}(\xi) e^{-\lambda_{1} \xi}=\lambda_{1} .
\end{gathered}
$$

Moreover, we have

$$
\begin{aligned}
u_{-}^{*} & =\lim _{\xi \rightarrow+\infty} \phi^{-}(\xi) \leq \liminf _{\xi \rightarrow+\infty} \phi(\xi):=l_{1} \leq \limsup _{\xi \rightarrow+\infty} \phi(\xi) \\
& :=l_{2} \leq \lim _{\xi \rightarrow+\infty} \bar{\phi}^{+}(\xi)=b .
\end{aligned}
$$


Next, we prove that

$$
l_{1}=l_{2}=K \quad \text { or } \quad u_{-}^{*} \leq l_{1}<K<l_{2} \leq b .
$$

In fact, if $l_{1}=l_{2}$, then $\lim _{\xi \rightarrow+\infty} \phi(\xi)=l_{1}$ exists. Taking $\xi \rightarrow$ $+\infty$ and applying L'Hospital's rule to (32), we can obtain $(a+$ $\alpha+\beta) f\left(l_{1}\right)=l_{1}$ which yields $l_{1}=l_{2}=K$.

Now let us consider $l_{1}<l_{2}$. Since it is impossible that $\phi(\xi)$ is eventually monotone, $\phi(\xi)$ is oscillating on positive infinity and then there exist two sequences $\left\{\zeta_{n}\right\}_{n \in \mathbb{N}}$ with $\zeta_{n} \rightarrow \infty$ as $n \rightarrow \infty$ and $\left\{\xi_{k}\right\}_{k \in \mathbb{N}}$ with $\xi_{k} \rightarrow \infty$ as $k \rightarrow \infty$ such that

$$
\begin{aligned}
& \phi\left(\zeta_{n}\right) \longrightarrow l_{1}, \quad \phi^{\prime}\left(\zeta_{n}\right) \longrightarrow 0 \text { as } n \longrightarrow \infty, \\
& \phi\left(\xi_{k}\right) \longrightarrow l_{2}, \quad \phi^{\prime}\left(\xi_{k}\right) \longrightarrow 0 \text { as } k \longrightarrow \infty .
\end{aligned}
$$

Since $f$ is continuous, for $\epsilon>0$, there exists a $\delta>0$ such that

$$
\begin{aligned}
& \max \left\{f(u) \mid u \in\left[l_{1}-\delta, l_{2}+\delta\right]\right\} \\
& \quad<\max \left\{f(u) \mid u \in\left[l_{1}, l_{2}\right]\right\}+\epsilon .
\end{aligned}
$$

For such a $\delta>0$, there exists a large enough number $M>0$ such that

$$
\phi(\xi) \in\left[l_{1}-\delta, l_{2}+\delta\right], \quad \forall \xi \geq M .
$$

If $\tau<+\infty$, we choose a large enough integer $N_{1}>0$ such that $\xi_{k}>M+m-c \tau$ for all $k>N_{1}$. Thus, for $\xi_{k}>M+m-c \tau$, we have

$$
\begin{aligned}
-c \phi^{\prime}\left(\xi_{k}\right)+\phi\left(\xi_{k}\right)= & \sum_{i=1}^{m} a_{i} \int_{0}^{\tau} J_{i}(y) f\left(\phi\left(\xi_{k}-i+c y\right)\right) d y \\
& +\alpha \int_{0}^{\tau} J_{m+1}(y) f\left(\phi\left(\xi_{k}+c y\right)\right) d y \\
& +\sum_{j=1}^{l} \beta_{j} \int_{0}^{\tau} J_{m+1+j}(y) \\
\leq & \left(\sum_{i=1}^{m} a_{i}+\alpha+\sum_{j=1}^{l} \beta_{j}\right) \\
& \times \max \left\{f(u) \mid u \in\left[l_{1}-\delta, l_{2}+\delta\right]\right\} \\
< & (a+\alpha+\beta) \\
& \times \max \left\{f(u) \mid u \in\left[l_{1}, l_{2}\right]\right\}+\epsilon .
\end{aligned}
$$

If $\tau=+\infty$, for the above $\epsilon>0$, there exists a sufficiently large number $M_{1}>0$ such that

$$
\begin{gathered}
\sum_{i=1}^{m} a_{i} \int_{M_{1}}^{+\infty} J_{i}(y) f\left(\phi\left(\xi_{k}-i+c y\right)\right) d y<\epsilon, \\
\alpha \int_{M_{1}}^{+\infty} J_{m+1}(y) f\left(\phi\left(\xi_{k}+c y\right)\right) d y<\epsilon, \\
\sum_{j=1}^{l} \beta_{j} \int_{M_{1}}^{+\infty} J_{m+1+j}(y) f\left(\phi\left(\xi_{k}+j+c y\right)\right) d y<\epsilon .
\end{gathered}
$$

Now let us choose a large enough integer $N_{2}>0$ such that $\xi_{k}>M+m-c M_{1}$ for all $k>N_{2}$. Therefore, for $\xi_{k}>M+$ $m-c M_{1}$, we have

$$
\begin{aligned}
-c \phi^{\prime}\left(\xi_{k}\right)+\phi\left(\xi_{k}\right)= & \sum_{i=1}^{m} a_{i} \int_{0}^{\tau} J_{i}(y) f\left(\phi\left(\xi_{k}-i+c y\right)\right) d y \\
& +\alpha \int_{0}^{\tau} J_{m+1}(y) f\left(\phi\left(\xi_{k}+c y\right)\right) d y \\
& +\sum_{j=1}^{l} \beta_{j} \int_{0}^{\tau} J_{m+1+j}(y) \\
< & 3 \epsilon+\left(\sum_{i=1}^{m} a_{i}+\alpha+\sum_{j=1}^{l} \beta_{j}\right) \\
& \times \max \left\{f(u) \mid u \in\left[l_{1}-\delta, l_{2}+\delta\right]\right\} \\
< & (a+\alpha+\beta)) d y \\
& \times \max \left\{f(u) \mid u \in\left[l_{1}, l_{2}\right]\right\}+4 \epsilon .
\end{aligned}
$$

According to the above argument and taking the limit as $k \rightarrow+\infty$, we obtain

$$
l_{2} \leq(a+\alpha+\beta) \max \left\{f(u) \mid u \in\left[l_{1}, l_{2}\right]\right\}+\epsilon .
$$

Since $\epsilon$ is arbitrary, it follows that

$$
l_{2} \leq(a+\alpha+\beta) \max \left\{f(u) \mid u \in\left[l_{1}, l_{2}\right]\right\} .
$$

Similarly, we can obtain

$$
l_{1} \geq(a+\alpha+\beta) \min \left\{f(u) \mid u \in\left[l_{1}, l_{2}\right]\right\} .
$$

If $l_{1}<l_{2} \leq K$, then (61) and (F2) imply that

$$
l_{1} \geq(a+\alpha+\beta) \min \left\{f(u) \mid l_{1} \leq u \leq l_{2}\right\}>l_{1},
$$

which leads to a contradiction. On the other hand, if $K \leq l_{1}<$ $l_{2}$, then (60) and (F2) also imply that

$$
l_{2} \leq(a+\alpha+\beta) \max \left\{f(u) \mid l_{1} \leq u \leq l_{2}\right\}<l_{2},
$$

which also gives a contradiction. Hence we conclude that $l_{1}<$ $K<l_{2}$.

If, in addition, (F4) holds, then we claim that $\lim _{\xi \rightarrow+\infty} \phi(\xi)=K$. Suppose the claim is false; that is, $l_{1}<l_{2}$. Let $k_{1}, k_{2} \in\left[l_{1}, l_{2}\right]$ such that

$$
\begin{aligned}
& f\left(k_{1}\right)=\max \left\{f(u) \mid l_{1} \leq u \leq l_{2}\right\}, \\
& f\left(k_{2}\right)=\min \left\{f(u) \mid l_{1} \leq u \leq l_{2}\right\} .
\end{aligned}
$$

Then we consider the following three cases.

Case 1. $K \leq k_{1} \leq l_{2}$.

If $k_{1}=l_{2}$, according to (60), we have $l_{2} \leq(a+\alpha+\beta) f\left(l_{2}\right)$ since $l_{2}>K$. Therefore $k_{1}<l_{2}$. Then, according to (60) and 
$k_{1} \geq K$, we have $l_{2} \leq(a+\alpha+\beta) f\left(k_{1}\right) \leq k_{1}<l_{2}$, which leads to a contradiction.

Case 2. $l_{1} \leq k_{2} \leq K$.

Similar to the discussion of Case 1, the case cannot happen.

Case 3. $k_{1}<K<k_{2}$.

By (60)-(61) and (F4), it follows that

$$
\begin{aligned}
l_{2}-l_{1} & \leq(a+\alpha+\beta) f\left(k_{1}\right)-(a+\alpha+\beta) f\left(k_{2}\right) \\
& <\left(2 K-k_{1}\right)-\left(2 K-k_{2}\right)=k_{2}-k_{1},
\end{aligned}
$$

which is impossible.

Thus, $l_{1}=l_{2}$; that is, the $\operatorname{limit}_{\lim _{\xi \rightarrow+\infty}} \phi(\xi)=l_{1} \in\left[u_{-}^{*}, b\right]$ exists. Taking $\xi \rightarrow+\infty$ and applying L'Hospital's rule to (32), we obtain that $l_{1}=l_{2}=K$. Hence, the assertion of part (i) follows.

(2) Since $f$ is an odd function, letting $\psi=-\phi$, then (13) reduces to

$$
\begin{aligned}
-c \psi^{\prime}(\xi)= & -\psi(\xi)+\sum_{i=1}^{m} a_{i} \int_{0}^{\tau} J_{i}(y) f(\psi(\xi-i+c y)) d y \\
& +\alpha \int_{0}^{\tau} J_{m+1}(y) f(\psi(\xi+c y)) d y \\
& +\sum_{j=1}^{l} \beta_{j} \int_{0}^{\tau} J_{m+1+j}(y) f(\psi(\xi+j+c y)) d y .
\end{aligned}
$$

It is easy to check that (66) has the same form as (13) and hence we can obtain the result of part (ii). The proof is complete.

Proof of Theorem 5. Let us assume that $\beta=0$ and $(a+$ $\alpha) f^{\prime}(0)>1$. Similar to the definitions of (36), for any $c<0$, we define the continuous functions as follows:

$$
\begin{array}{r}
\bar{\phi}^{+}(\xi)=: \min \left\{b, e^{\lambda_{3} \xi}\right\}, \quad \bar{\phi}^{-}(\xi)=: \min \left\{u_{-}^{*}, e^{\lambda_{3} \xi}\right\} \\
\text { for } \xi \in \mathbb{R},
\end{array}
$$

where $\lambda_{3}$ is defined in Lemma 11. Similar to the proofs of Lemmas 14-16, it is easily seen that $T(\Gamma) \subset \Gamma$ and $T: \Gamma \rightarrow \Gamma$ is compact with respect to the norm $\|\cdot\|_{X_{\lambda}}$ in $X_{\lambda}$, where $\lambda \epsilon$ $\left(0, \lambda_{3}\right)$. Hence, by the same arguments as those of the proof of Theorem 3, we can obtain the assertions of the theorem. This completes the proof.

4.2. Traveling Waves with (BC2) and (BC4). By arguments similar to those of the proof of Theorem 3, in this section we show the existence of traveling wave solutions satisfying conditions (BC2) and (BC4).

For $\gamma>1 / c>0$, we define the operator $\bar{T}$ : $C(\mathbb{R},[0, b]) \rightarrow C(\mathbb{R},[0, b])$ by

$$
\bar{T}(\phi)(\xi)=-e^{\gamma \xi} \int_{\xi}^{\infty} e^{-\gamma y} H(\phi)(y) d y
$$

where

$$
\begin{aligned}
H(\phi)(\xi)= & \left(\frac{1}{c}-\gamma\right) \phi(\xi) \\
& -\frac{1}{c} \sum_{i=1}^{m} a_{i} \int_{0}^{\tau} J_{i}(y) f(\phi(\xi-i+c y)) d y \\
& -\frac{\alpha}{c} \int_{0}^{\tau} J_{m+1}(y) f(\phi(\xi+c y)) d y \\
& -\frac{1}{c} \sum_{j=1}^{l} \int_{0}^{\tau} J_{m+1+j}(y) f(\phi(\xi+j+c y)) d y .
\end{aligned}
$$

According to (F1) and (J), $\bar{T}$ is well defined. It is obvious that a fixed point $\phi$ of $\bar{T}$ is a traveling wave solution of (5). Similar to (33), we may define $\bar{T}^{ \pm}$through $f^{ \pm}$in the same way.

First, we consider $a>0$ and $(a+\alpha) f^{\prime}(0)>1$. Let us define the continuous functions $\bar{w}^{ \pm}(\xi)$ by

$$
\begin{array}{r}
\bar{w}^{+}(\xi):=\min \left\{b, e^{\lambda_{2} \xi}\right\}, \quad \bar{w}^{-}(\xi):=\min \left\{u_{-}^{*}, e^{\lambda_{2} \xi}\right\}, \\
\xi \in \mathbb{R} .
\end{array}
$$

Since $f^{-}$is nondecreasing and satisfies the assumptions illustrated in [26], similar to Theorem 1.2 of [26], we can obtain the following results.

Lemma 17. Assume that (F1)-(F3) hold. Then, for any $c>c_{2}^{*}$, one has

$$
\bar{T}^{+}\left(\bar{w}^{+}\right)(\xi) \leq \bar{w}^{+}(\xi), \quad \bar{T}^{-}\left(\bar{w}^{-}\right)(\xi) \leq \bar{w}^{-}(\xi) \quad \forall \xi \in \mathbb{R} .
$$

Lemma 18. Assume that (F1)-(F3) hold. Then, for any $c>c_{2}^{*}$, there exists a fixed point $w^{-}$of $\bar{T}^{-}$such that $\bar{T}^{-}\left(w^{-}\right)(\xi)=w^{-}(\xi)$ and $w^{-}(\xi) \leq \bar{w}^{-}(\xi)$ for all $\xi \in \mathbb{R}$. Moreover,

$$
\lim _{\xi \rightarrow+\infty} w^{-}(\xi) e^{-\lambda_{2} \xi}=1, \quad \lim _{\xi \rightarrow-\infty} w^{-}(\xi)=u_{-}^{*} .
$$

Now, fixing a number $\lambda \in\left(\lambda_{2}, 0\right)$, we define the set $\bar{\Gamma}$ by

$$
\bar{\Gamma}:=\left\{\phi \in X_{\lambda} \mid w^{-}(\xi) \leq \phi(\xi) \leq \bar{w}^{+}(\xi), \text { for } \xi \in \mathbb{R}\right\} .
$$

It is easy to see that $\bar{\Gamma}$ is a nonempty, closed, and convex subset of $X_{\lambda}$. Similar to the proof of Lemma 16, we have the following lemma.

Lemma 19. Assume $a>0$ and $(a+\alpha) f^{\prime}(0)>1$. The following assertions hold.

(1) $\bar{T}(\bar{\Gamma}) \subset \bar{\Gamma}$;

(2) $\bar{T}: \bar{\Gamma} \rightarrow \bar{\Gamma}$ is compact with respect to the norm $\|\cdot\|_{X_{\lambda}}$ in $X_{\lambda}$.

Now we start the proofs of Theorems 4 and 6 . 
Proof of Theorem 4. According to Lemma 19 and Schauder's fixed point theorem, there exists $\phi \in \bar{\Gamma}$ such that $\phi=\bar{T}(\phi)$. Since $0 \leq w^{-}(\xi) \leq \phi(\xi) \leq \bar{w}^{+}(\xi) \leq b$ for $\xi \in \mathbb{R}$ and $\lambda_{2}<0$, we can easily obtain that, for $c>c_{2}^{*}$,

$$
\begin{gathered}
\lim _{\xi \rightarrow+\infty} \phi(\xi)=0, \\
\lim _{\xi \rightarrow+\infty} \phi(\xi) e^{-\lambda_{2} \xi}=1, \\
\lim _{\xi \rightarrow+\infty} \phi^{\prime}(\xi) e^{-\lambda_{2} \xi}=\lambda_{2} .
\end{gathered}
$$

By the same arguments as those of the proof of Theorem 3, we obtain the assertions of the theorem. This completes the proof.

Next, we consider the case $a=0$ and $(\alpha+\beta) f^{\prime}(0)>1$. For any $c>0$, let us redefine the continuous functions $\bar{w}^{ \pm}(\xi)$ by

$$
\begin{array}{r}
\bar{w}^{+}(\xi):=\min \left\{b, e^{\lambda_{4} \xi}\right\}, \quad \bar{w}^{-}(\xi):=\min \left\{u_{-}^{*}, e^{\lambda_{4} \xi}\right\}, \\
\xi \in \mathbb{R} .
\end{array}
$$

Then, similar to that of Theorem 5, we obtain the conclusion of Theorem 6.

\section{Conclusion}

In this paper, the motivation of our work is to consider the existence of traveling wave solutions for DCNN model with nonmonotonic output functions. We have developed a technique to construct two appropriate nondecreasing functions to squeeze the nonmonotonic output functions. By adopting Schauder's fixed point theorem, the existence of traveling wave solutions has been derived.

\section{Conflict of Interests}

The authors declare that there is no conflict of interests regarding the publication of this paper.

\section{Acknowledgments}

Zhi-Xian Yu is partially supported by the National Natural Science Foundation of China, Shanghai Leading Academic Discipline Project (no. XTKX2012), Innovation Program of Shanghai Municipal Education Commission (no. 14YZ096), and Hujiang Foundation of China (B14005). Rong Yuan and Ming-Shu Peng are partially supported by the National Natural Science Foundation of China and RFDP. ChengHsiung Hsu is partially supported by the National Science Council and NCTS of Taiwan.

\section{References}

[1] C. H. Hsu, C. H. Li, and S. Y. Yang, "Diversity of traveling wave solutions in delayed cellular neural networks," International Journal of Bifurcation and Chaos in Applied Sciences and Engineering, vol. 18, no. 12, pp. 3515-3550, 2008.
[2] C. Hsu and S. Lin, "Existence and multiplicity of traveling waves in a lattice dynamical system," Journal of Differential Equations, vol. 164, no. 2, pp. 431-450, 2000.

[3] C.-H. Hsu, S.-S. Lin, and W. Shen, "Traveling waves in cellular neural networks," International Journal of Bifurcation and Chaos, vol. 9, no. 7, pp. 1307-1319, 1999.

[4] R. Ponalagusamy and S. Senthilkumar, "Investigation on raster CNN simulation by numerical integration algorithms," Journal of Combinatorial Mathematics and Combinatorial Computing, vol. 67, pp. 35-48, 2008.

[5] R. Ponalagusamy and S. Senthilkumar, "A new fourth order embedded RKAHeM(4,4) method with error control on multilayer raster cellular neural network," Signal, Image and Video Processing, vol. 3, no. 1, pp. 1-11, 2009.

[6] R. Ponalagusamy and S. Senthilkumar, "Investigation on timemultiplexing cellular neural network simulation by RKA$\operatorname{HeM}(4,4)$ technique," International Journal of Advanced Intelligence Paradigms, vol. 3, no. 1, pp. 43-66, 2011.

[7] L. O. Chua, CNN: A Paradigm for Complexity, vol. 31 of World Scientific Series on Nonlinear Science, Series A, World Scientific, Singapore, 1998.

[8] L. O. Chua and L. Yang, "Cellular neural networks: theory," IEEE Transactions on Circuits and Systems, vol. 35, no. 10, pp. 1257$1272,1988$.

[9] L. O. Chua and L. Yang, "Cellular neural networks: applications," IEEE Transactions on Circuits and Systems, vol. 35, no. 10, pp. 1273-1290, 1988.

[10] X. Liu, P. Weng, and Z. Xu, "Existence of traveling wave solutions in nonlinear delayed cellular neural networks," Nonlinear Analysis: Real World Applications, vol. 10, no. 1, pp. 277-286, 2009.

[11] P. X. Weng and J. Wu, "Deformation of traveling waves in delayed cellular neural networks," International Journal of Bifurcation and Chaos in Applied Sciences and Engineering, vol. 13, no. 4, pp. 797-813, 2003.

[12] C.-H. Hsu and S.-Y. Yang, "Structure of a class of traveling waves in delayed cellular neural networks," Discrete and Continuous Dynamical Systems A, vol. 13, no. 2, pp. 339-359, 2005.

[13] C.-H. Hsu and S.-Y. Yang, “Traveling wave solutions in cellular neural networks with multiple time delays," Discrete and Continuous Dynamical Systems A, pp. 410-419, 2005.

[14] S. Ma, "Traveling waves for non-local delayed diffusion equations via auxiliary equations," Journal of Differential Equations, vol. 237, no. 2, pp. 259-277, 2007.

[15] M. Mei, C.-K. Lin, C.-T. Lin, and J. So, "Traveling wavefronts for time-delayed reaction-diffusion equation. I. Local nonlinearity," Journal of Differential Equations, vol. 247, no. 2, pp. 495510, 2009.

[16] M. Mei, C. K. Lin, C. T. Lin, and W. H. So, “Traveling wavefronts for time-delayed reaction-diffusion equation: (II) Nonlocal nonlinearity," Journal of Differential Equations, vol. 247, no. 2, pp. 511-529, 2009.

[17] K. W. Schaaf, "Asymptotic behavior and traveling wave solutions for parabolic functional-differential equations," Transactions of the American Mathematical Society, vol. 302, no. 2, pp. 587-615, 1987.

[18] A. I. Volpert, V. A. Volpert, and V. Volpert, Traveling Wave Solutions of Parabolic Systems, vol. 140 of Translations of Mathematical Monographs, American Mathematical Society, Providence, RI, USA, 1994. 
[19] H. Wang, "On the existence of traveling waves for delayed reaction-diffusion equations," Journal of Differential Equations, vol. 247, no. 3, pp. 887-905, 2009.

[20] J. Wu and X. Zou, "Traveling wave front solutions in reactiondiffusion systems with delay," Journal of Differential Equations, vol. 13, pp. 651-687, 2001.

[21] S. Wu and H. Zhao, "Traveling fronts for a delayed reactiondiffusion system with a quiescent stage," Communications in Nonlinear Science and Numerical Simulation, vol. 16, no. 9, pp. 3610-3621, 2011.

[22] Z. Yu and R. Yuan, "Traveling wave fronts in reaction-diffusion systems with spatio-temporal delay and applications," Discrete and Continuous Dynamical Systems B, vol. 13, no. 3, pp. 709728, 2010.

[23] Z. Yu and R. Yuan, "Traveling waves of delayed reactiondiffusion systems with applications," Nonlinear Analysis: Real World Applications, vol. 12, no. 5, pp. 2475-2488, 2011.

[24] J. Mallet-Paret, "The global structure of traveling waves in spatially discrete dynamical systems," Journal of Dynamics and Differential Equations, vol. 11, no. 1, pp. 49-127, 1999.

[25] S.-N. Chow, J. Mallet-Paret, and W. Shen, "Traveling waves in lattice dynamical systems," Journal of Differential Equations, vol. 149, no. 2, pp. 248-291, 1998.

[26] Z. X. Yu, R. Yuan, C. H. Hsu, and Q. Jiang, "Traveling waves for nonlinear cellular neural networks with distributed delays," Journal of Differential Equations, vol. 251, no. 3, pp. 630-650, 2011.

[27] C. Hsu and S. Yang, "On camel-like traveling wave solutions in cellular neural networks," Journal of Differential Equations, vol. 196, no. 2, pp. 481-514, 2004. 


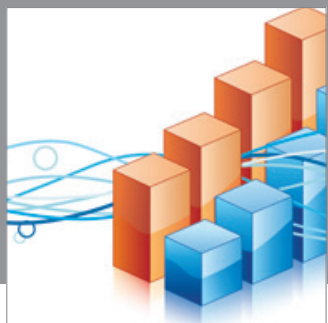

Advances in

Operations Research

mansans

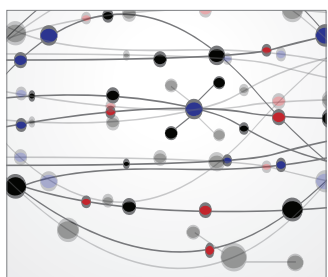

The Scientific World Journal
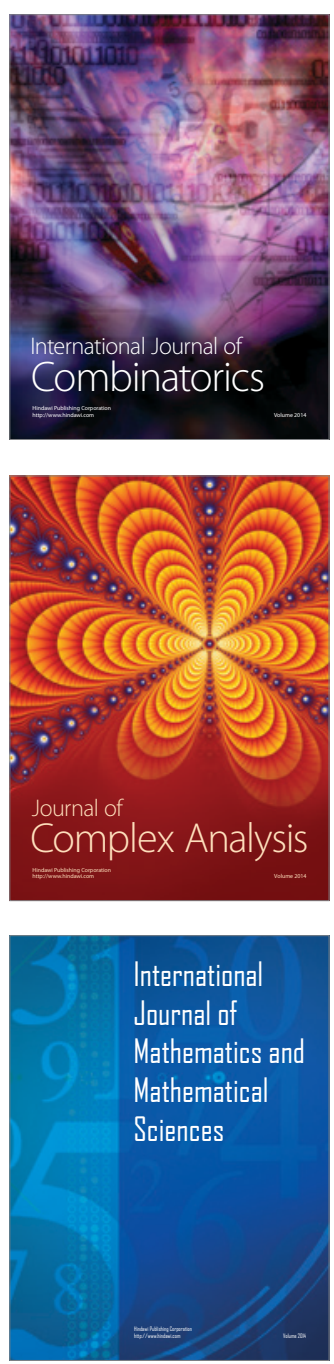
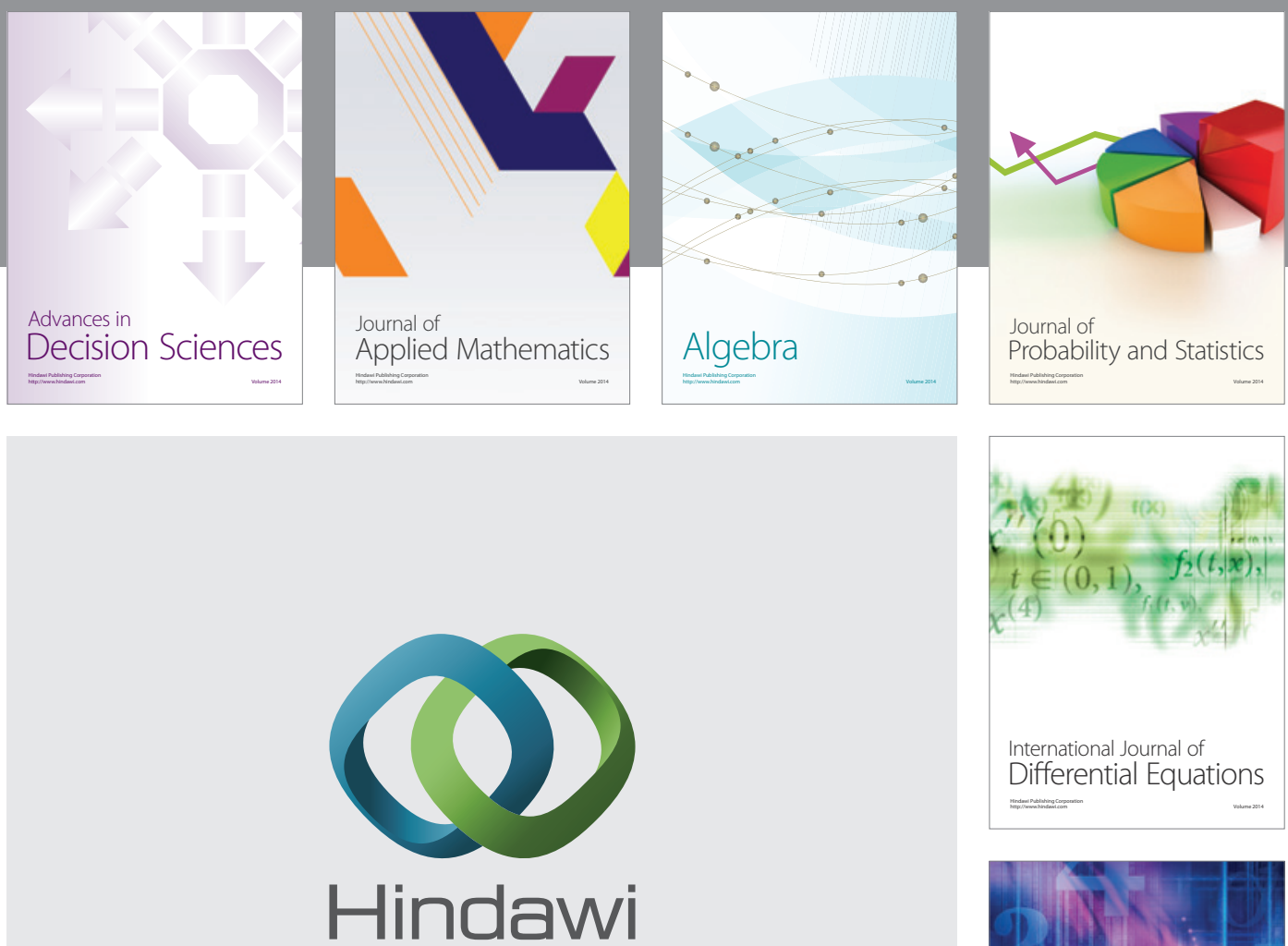

Submit your manuscripts at http://www.hindawi.com
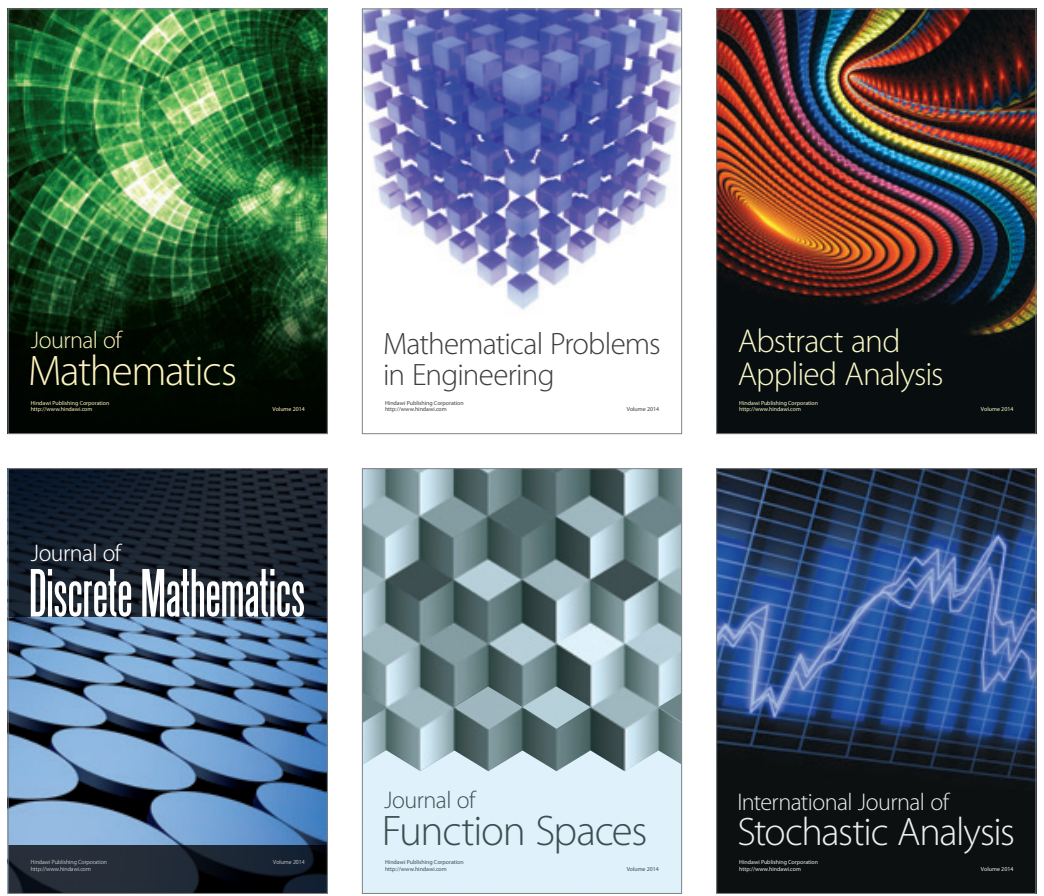

Journal of

Function Spaces

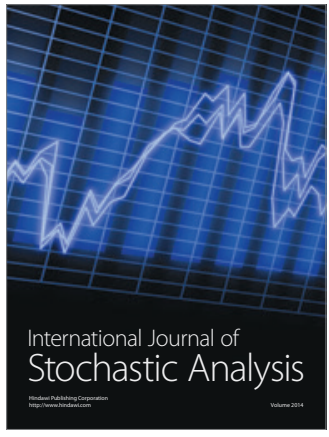

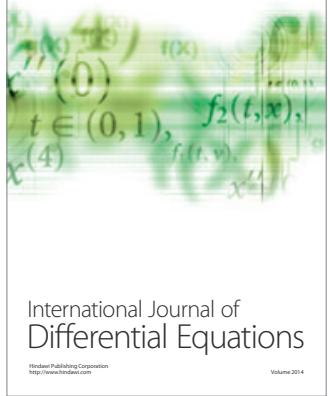
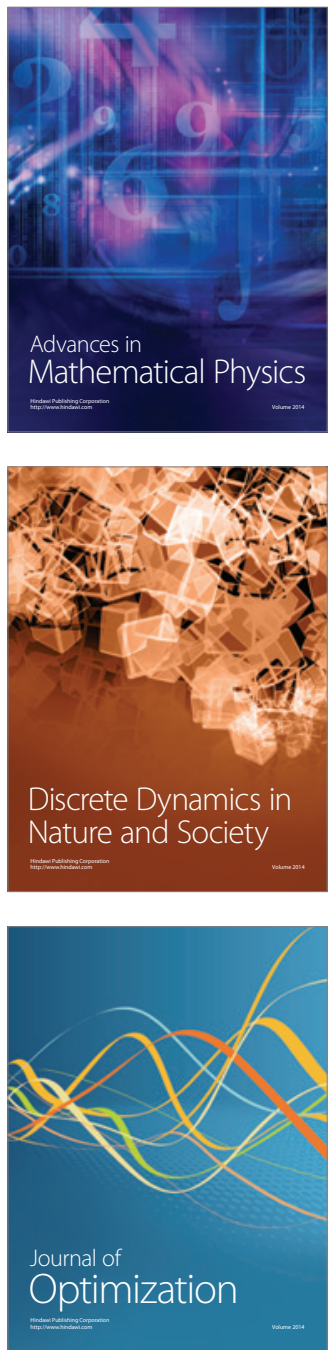